OPEN ACCESS

Edited by:

Jun Aruga,

Nagasaki University, Japan

Reviewed by:

Daniel Vogt,

Michigan State University,

United States

Goichi Miyoshi,

Tokyo Women's Medical University, Japan

*Correspondence:

Zhejun Xu

14111520028@fudan.edu.cn

Yan You

youyan@fudan.edu.cn

${ }^{\dagger}$ These authors have contributed equally to this work

Received: 24 January 2019 Accepted: 11 March 2019

Published: 02 April 2019

Citation:

Tao G, Li Z, Wen Y, Song X, Wei S, $D u H$, Yang $Z, X u Z$ and You $Y$ (2019) Transcription Factors Sp8 and

Sp9 Regulate Medial Ganglionic

Eminence-Derived Cortical

Interneuron Migration.

Front. Mol. Neurosci. 12:75. doi: 10.3389/fnmol.2019.00075

\section{Transcription Factors Sp8 and Sp9 Regulate Medial Ganglionic Eminence-Derived Cortical Interneuron Migration}

\author{
Guangxu Tao ${ }^{\dagger}$, Zhenmeiyu $\mathrm{Li}^{\dagger}$, Yan Wen, Xiaolei Song, Song Wei, Heng Du, \\ Zhengang Yang, Zhejun $\mathrm{Xu}$ *and Yan You*
}

State Key Laboratory of Medical Neurobiology, MOE Frontier Research Center for Brain Science, Department of Neurology, Institutes of Brain Science, Zhongshan Hospital, Fudan University, Shanghai, China

Cortical interneurons are derived from the subpallium and reach the developing cortex through long tangential migration. Mature cortical interneurons are characterized by remarkable morphological, molecular, and functional diversity. The calcium-binding protein parvalbumin (PV) and neuropeptide somatostatin (SST) identify most medial ganglionic eminence (MGE)-derived cortical interneurons. Previously, we demonstrated that Sp9 plays a curial transcriptional role in regulating MGE-derived cortical interneuron development. Here, we show that SP8 protein is weekly expressed in the MGE mantle zone of wild type mice but upregulated in Sp9 null mutants. $\mathrm{PV}^{+}$cortical interneurons were severely lost in Sp8/Sp9 double conditional knockouts due to defects in tangential migration compared with Sp9 single mutants, suggesting that Sp8/9 coordinately regulate $\mathrm{PV}^{+}$cortical interneuron development. We provide evidence that Sp8/Sp9 activity is required for normal MGE-derived cortical interneuron migration, at least in part, through regulating the expression of EphA3, Ppp2r2c, and Rasgef1b.

Keywords: Sp8, Sp9, medial ganglionic eminence, cortical interneuron, tangential migration, parvalbumin, somatostatin

\section{INTRODUCTION}

The cerebral cortex plays an irreplaceable role in many advanced functions, such as learning, movement, emotion memory and decision-making. The normal execution of these functions depends on the subtle ratio of excitatory projection neurons to inhibitory interneurons in the cerebral cortex and the appropriate functional circuits formed between them (Rubenstein and Merzenich, 2003; Del Pino et al., 2018; Lim et al., 2018). Glutamatergic cortical excitatory projection neurons are generated by pallial (cortical) neuroepithelial and radial glial cells (Kriegstein and Alvarez-Buylla, 2009). On the other hand, GABAergic cortical inhibitory interneurons, both in primates and rodents, are derived from the subpallium and reach their final position in the cortex through tangential migration (Wonders and Anderson, 2006; Hansen et al., 2013; Ma et al., 2013). During development, the subpallium is composed of four major proliferative zones: the medial (MGE), lateral (LGE) and caudal (CGE) ganglionic eminences and the preoptic area (POA); the MGE generates $60 \%$ of all cortical interneurons and includes parvalbumin $\left(\mathrm{PV}^{+}\right)$and somatostatin $\left(\mathrm{SST}^{+}\right)$subtypes (Gelman and Marín, 2010; Hu et al., 2017; Wamsley and Fishell, 2017). 
The $S p 9$ zinc finger transcription factor is widely expressed in the ganglionic eminences (Long et al., 2009; Zhang et al., 2016). Sp8 and Sp9 are two closely related button head-like transcription factors and have many redundant functions in regulating GABAergic neuronal development (Li et al., 2018; $\mathrm{Xu}$ et al., 2018). Previously, we showed that $S p 9$ has a curial transcriptional role in regulating MGE-derived cortical interneuron development (Liu et al., 2018). While Sp8 is highly expressed in the dorsal LGE and the CGE (Ma et al., 2012), it is also weekly expressed in the MGE (Vogt et al., 2014). However, the function of $S p 8$ in the MGE remains largely unknown.

In the present study, we found that SP8 protein expression was upregulated in the MGE mantle zone of $S p 9$ null mutants. There were more MGE-derived cortical interneurons that failed to migrate into the cortex in $S p 8 / S p 9$ double conditional knockouts than in $S p 9$ single conditional knockouts. Furthermore, fewer $\mathrm{Erbb}^{+}$cells (immature $\mathrm{PV}^{+}$cortical interneurons) migrated in the cortical marginal zone (MZ), whereas more migrated in the cortical subventricular zone (SVZ). In the postnatal cortex, the most prominent phenotype was that approximately $80 \%$ of $\mathrm{PV}^{+}$interneurons were lost in $S p 8 / S p 9$ double conditional knockouts. We provide evidence that $S p 8$ and $S p 9$ mediate these effects by regulating the expression of several genes that regulate cortical interneuron migration and development, such as EphA3, Ppp2r2c and, Rasgef $1 b$.

\section{MATERIALS AND METHODS}

\section{Mice}

All animal experiments described in this study were approved in accordance with institutional guidelines, and the institutional review board (Ethics Committee) of Shanghai Medical College of Fudan University approved the study design. The strains used in this study have been previously reported: Nkx2-1Cre (Xu et al., 2008), Rosa26-YFP (R26R-YFP; Srinivas et al., 2001), Sp $9^{\text {LacZ/+ }}$ (Zhang et al., 2016), Sp $9^{F /+}$ (Zhang et al., 2016), $S p 8^{F /+}$ (Bell et al., 2003) and Lhx6-Cre (Fogarty et al., 2007). All lines were maintained in a mixed genetic background of C57BL/6J, 129 and CD1. Pregnancy of mated mice was determined by vaginal plug detection, which was defined as embryonic day 0.5 (E0.5), and the day of birth was defined as postnatal day 0 (P0). The range of embryos used had a \pm 0.5 day deviation.

\section{Tissue Preparation}

The pregnant mice were killed by cervical dislocation on designated dates. Each embryo was separated from the placenta, and the brain was dissected out and then fixed in $4 \%$ diethylpyrocarbonate and paraformaldehyde (DEPC-PFA) for several hours or overnight. Postnatal mice were deeply anesthetized and perfused adequately with cold $0.01 \mathrm{M} \mathrm{PBS}$ and $4 \%$ DEPC-PFA, and brains were then removed and postfixed overnight. Cryosectioning (Leica, CM 1950) was performed on brains embedded in optimal cutting temperature (OCT; Sakura); brains were immersed in OCT for $5 \mathrm{~min}$ at $4^{\circ} \mathrm{C}$ and subsequently transferred to a plastic mold filled with OCT and frozen in dry ice-chilled ethanol.

\section{Immunohistochemistry}

Immunohistochemistry was performed on $12 \mu \mathrm{m}$ (embryonic brains) or $30 \mu \mathrm{m}$ (postnatal brains) coronal sections (Liu et al., 2018). Briefly, sections were blocked for $30 \mathrm{~min}$ in TBS with $0.1 \%$ Triton X-100 and 5\% donkey serum. For double staining, sections were incubated simultaneously with primary antibodies from different species, and secondary antibodies were used sequentially. Primary antibodies were incubated for $24 \mathrm{~h}$ at $4^{\circ} \mathrm{C}$. We used rabbit anti-calretinin (CR; 1:3,000, AB5054, Millipore, Burlington, MA, USA), chicken anti-GFP (1:2,000, GFP-1020, Aves Labs), rabbit anti-PV (1:2,000, PV25, Swant), rabbit antiNKX2-1 (1:500, sc-13040, Santa Cruz Biotechnology, Dallas, TX, USA), rabbit anti-NPY (1:500, 22940, Incstar), goat anti-SST (1:500, sc-7819, Santa Cruz Biotechnology, Dallas, TX, USA) and goat anti-SP8 (1:2,000, sc-104661, Santa Cruz Biotechnology, Dallas, TX, USA). Secondary antibodies (1:400, Jackson Immuno Research) were incubated for $2 \mathrm{~h}$ at room temperature (RT), rinsed three times in TBS, and then incubated with DAPI $\left(4^{\prime}, 6\right.$ diamidino-2-phenylindole, 1:5,000) for $3 \mathrm{~min}$.

\section{In situ Hybridization}

RNA in situ hybridization experiments were performed using digoxigenin riboprobes on $20 \mu \mathrm{m}$ cryostat sections (Liu et al., 2018; Xu et al., 2018). The previously described full-length cDNA probe of SST was used (McKinsey et al., 2013). Templates for making other riboprobes were amplified by PCR using the following primers:

\section{(1) Erbb4 Fwd: GCACCGATATTTGCCCCAA} Rev: CAGTCATGACTAGTGGGACCGTTAC

(2) EphA3 Fwd: TGTATGGAGTTACGGGATTGTTC

(3) $P p p 2 r 2 \mathrm{c}$ Rev: GGCTCTACACTAGTTCTTCCACTTC Fwd: CGGACGACCTACGCATCAACCT Rev: GCCCTGCCTCACGATTAACCCTA

(4) Rasgef1b Fwd: GTGGCTACAACCGAAACCTCTA Rev: AGACCGGGCTCATATTCATACC (5) Npas1 Fwd: CGTGCGTCTTAGCGTCACCTACC Rev: GCCTCCACTTTGATGCGTTTGC

\section{Microscopic Imaging}

Immunofluorescence staining images were taken using an Olympus BX51 metallographic microscope, an Olympus VS120 digital slice scanning system or an Olympus FV1000 laser scanning confocal microscope. Brightfield images (in situ hybridization) were taken using an Olympus BX51 metallographic microscope. Images were manipulated with Photoshop CS5 software (Adobe Systems, San Jose, CA, USA). The contrast and brightness of these images were adjusted for better visualization.

\section{Cell Counting and Data Collection}

DAPI staining was used to demarcate MZ, cortical plate (CP), subplate (SP), intermediate zone (IZ), SVZ, and VZ. The numbers of $\mathrm{GFP}^{+}$cells in the E15.5 cortex were counted in 

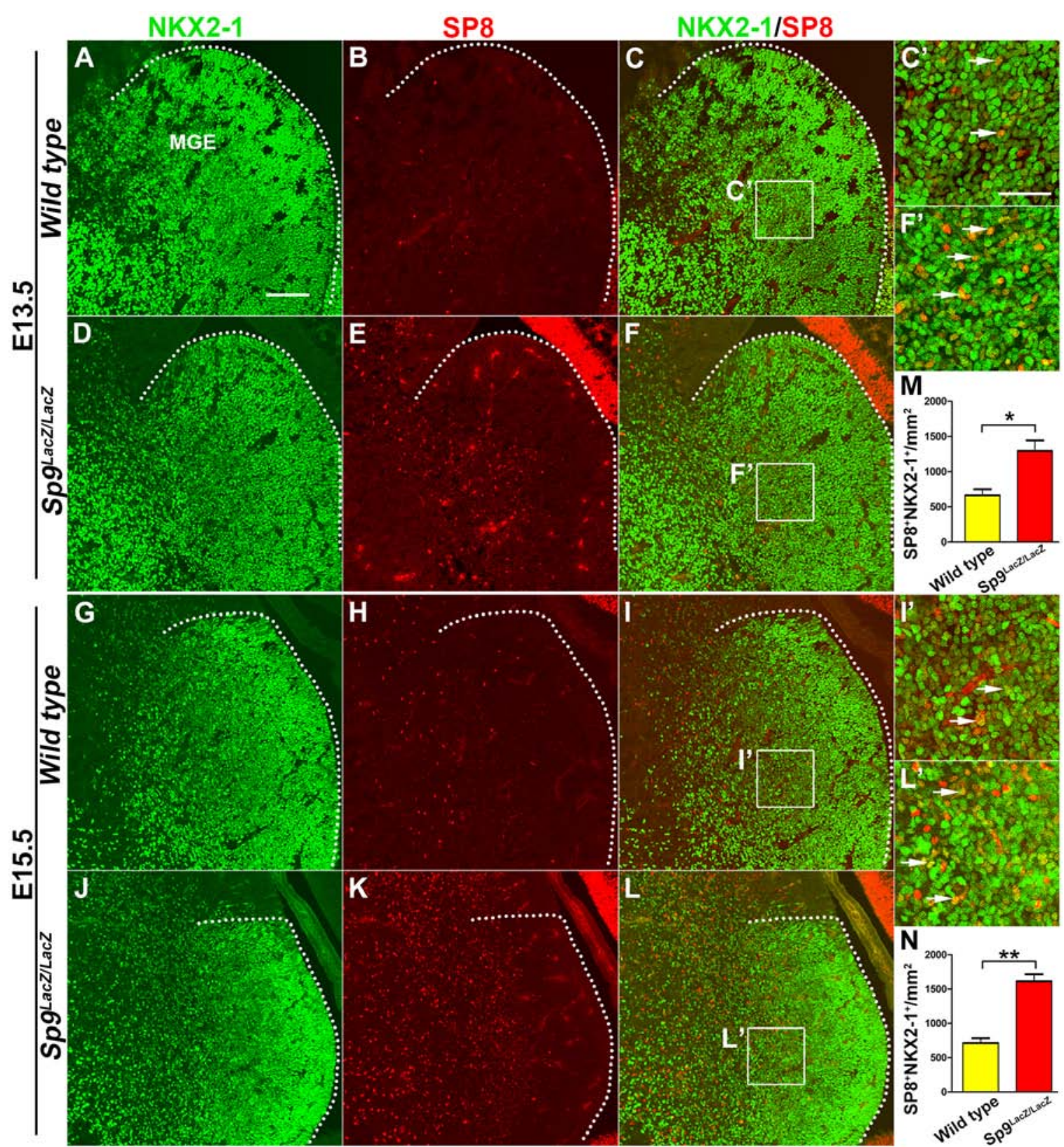

FIGURE 1 | SP8 expression is upregulated in medial ganglionic eminence (MGE) of SpgLacZ/LacZ null mutants. (A-L) NKX2-1/SP8 double-immunostained coronal sections at E13.5 and E15.5. ( $\left.\mathbf{C}^{\prime}, \mathbf{F}^{\prime}, \mathbf{I}^{\prime}, \mathbf{L}^{\prime}\right)$ Higher magnification images of the boxed areas in (C,F, I, L). Upregulation of SP8 protein expression can be noted in the mutant MGE mantle zone; most of these SP8 ${ }^{+}$cells exhibit NKX2-1 coexpression. (M,N) Quantified data showed that the density of SP8 ${ }^{+} / \mathrm{NKX2-1^{+ }}$ cells was increased in SpgLacZ/LacZ mutants compared with controls in E13.5 and E15.5. Scale bars: $100 \mu \mathrm{m}$ in (A) for (A-L); $50 \mu \mathrm{m}$ in $\left(\mathbf{C}^{\prime}\right)$ for $\left(\mathbf{C}^{\prime}, \mathbf{F}^{\prime}, \mathbf{I}^{\prime}, \mathbf{L}^{\prime}\right)$.

250- $\mu \mathrm{m}$-wide bins that spanned from the MZ to the VZ. The percentage of $\mathrm{GFP}^{+}$cells in each layer was calculated. And the numbers of Sst ${ }^{+}$, Erbb4 $^{+}$, Npasi ${ }^{+}$cells in the E15.5 cortex were counted in $350-\mu \mathrm{m}$-wide bins that spanned from the MZ to the VZ. We counted cells from three sections of each mouse and analyzed three mice of each genotype [Nkx2-1-Cre; Rosa-YFP (controls), Nkx2-1-Cre; Sp $9^{F / F}$; Rosa-YFP (Nkx2-1Cre; Sp9-CKOs); Nkx2-1-Cre; Sp $8^{F / F} ; S p 9^{F / F}$; Rosa-YFP (Nkx21-Cre; Sp8/9-DCKOs)]. For P30 mice, the numbers of $\mathrm{GFP}^{+}$,
$\mathrm{GFP}^{+} / \mathrm{PV}^{+}, \mathrm{GFP}^{+} / \mathrm{SST}^{+}, \mathrm{GFP}^{+} / \mathrm{NPY}^{+}$, and $\mathrm{GFP}^{+} / \mathrm{CR}^{+}$cells in the somatosensory cortex were analyzed in $1,000-\mu \mathrm{m}$-wide bins ( $n=3$ mice for each genotype, three sections per mouse).

IBM SPSS Statistic 22 and GraphPad Prism 6 was used for statistical analysis, and all data are expressed as the means \pm standard error. For comparisons of means between groups, $t$-test, One-way analysis of variance (ANOVA), the least significant difference (LSD) test, the Bonferroni correction, the Student-Newman-Keuls (SNK) test, Tamhane's T2 test, and 

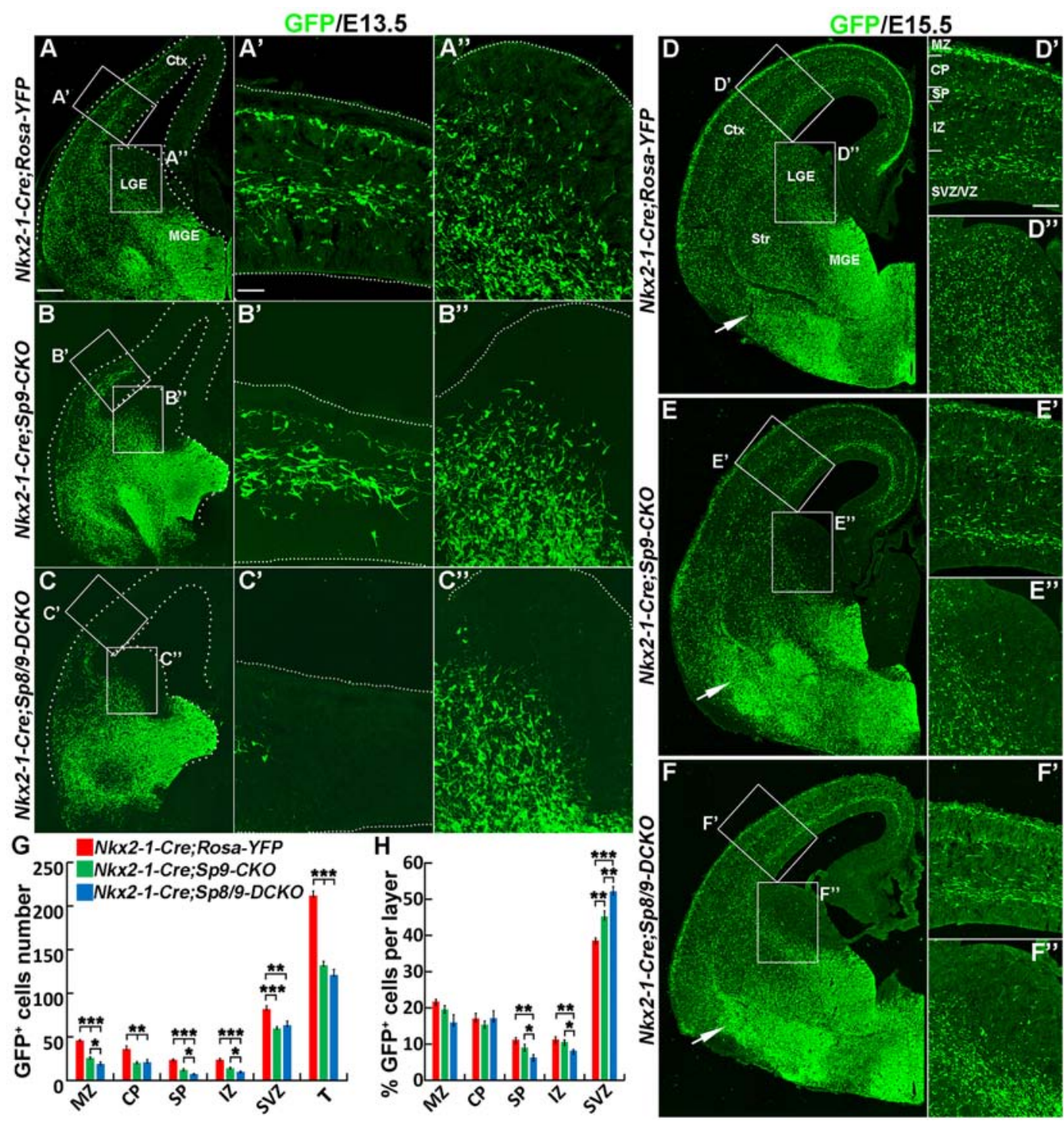

FIGURE 2 | Tangential migration defects of MGE-derived cortical interneurons in Nkx2-1-Cre; Sp8/9-DCKO mice. (A-C) GFP immunostained E13.5 coronal hemisections. $\left(\mathbf{A}^{\prime} \mathbf{-} \mathbf{C}^{\prime \prime}\right)$ Higher magnification images of the boxed areas in $\mathbf{( A - C )}$ show that MGE-derived GFP+ ${ }^{+}$cortical interneurons migrate to the cortex much less efficiently in Nkx2-1-Cre; Sp8/9-DCKO (double mutant) mice than in controls and Nkx2-1-Cre; Sp9-CKO (single mutant) mice. Very few GFP+ cells can be noted in

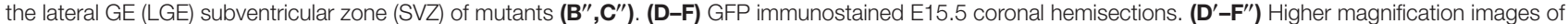
the boxed areas in (D-F). Note that more GFP+ cells ectopically accumulated in the ventral telencephalon in Nkx2-1-Cre; Sp8/9-DCKO mice than in controls and Nkx2-1-Cre; Sp9-CKO mice (arrows). (G,H) Quantification showing that mutant mice had fewer GFP ${ }^{+}$cells in the cortex at E15.5. The percentage of GFP+ cells was reduced in the SP and the intermediate zone (IZ) and increased in the SVZ. Ctx, cortex. ${ }^{*} P<0.05 ;{ }^{* *} P<0.01 ;{ }^{* * *} P<0.001$. Scale bars: $100 \mu \mathrm{m}$ in (A) for (A-F); 25 $\mu \mathrm{m}$ in $\left(\mathbf{A}^{\prime}\right)$ for $\left(\mathbf{A}^{\prime}-\mathbf{C}^{\prime \prime}\right) ; 50 \mu \mathrm{m}$ in $\left(\mathbf{D}^{\prime}\right)$ for $\left(\mathbf{D}^{\prime}-\mathbf{F}^{\prime \prime}\right)$

Dunnett's T3 test were used; $P<0.05$ was considered significant $\left({ }^{*} P<0.05\right.$; ${ }^{* *} P<0.01$; $\left.{ }^{* * *} P<0.001\right)$.

\section{RESULTS}

\section{SP8 Expression Is Upregulated in the MGE Mantle Zone of Sp9 Null Mutants}

SP9 is strongly expressed in the SVZ of the MGE, but very few SP9 ${ }^{+}$cells are observed in the MGE VZ (Liu et al., 2018).
SP8 is also not expressed in the MGE VZ and SVZ, but we observed that a subset of cells in the MGE mantle zone weakly expressed SP8 protein at E13.5 and E15.5 (Figures 1A-C',G-I'). This finding is consistent with a previous report, which showed that Sp8 mRNA is weakly expressed in the MGE (Vogt et al., 2014). In the Sp9 $9^{L a c Z / L a c Z}$ null mutant, however, SP8 expression was greatly upregulated in the MGE mantle zone and in a subset of MGE-derived cells (Figures $1 \mathbf{D}-\mathbf{F}^{\prime}, \mathbf{J}-\mathbf{L}^{\prime}$; Liu et al., 2018). We next quantified the number of NKX2-1 $1^{+} / \mathrm{SP}^{+}$cells in the MGE mantle zone. The Sp9 mutants showed $>120 \%$ 


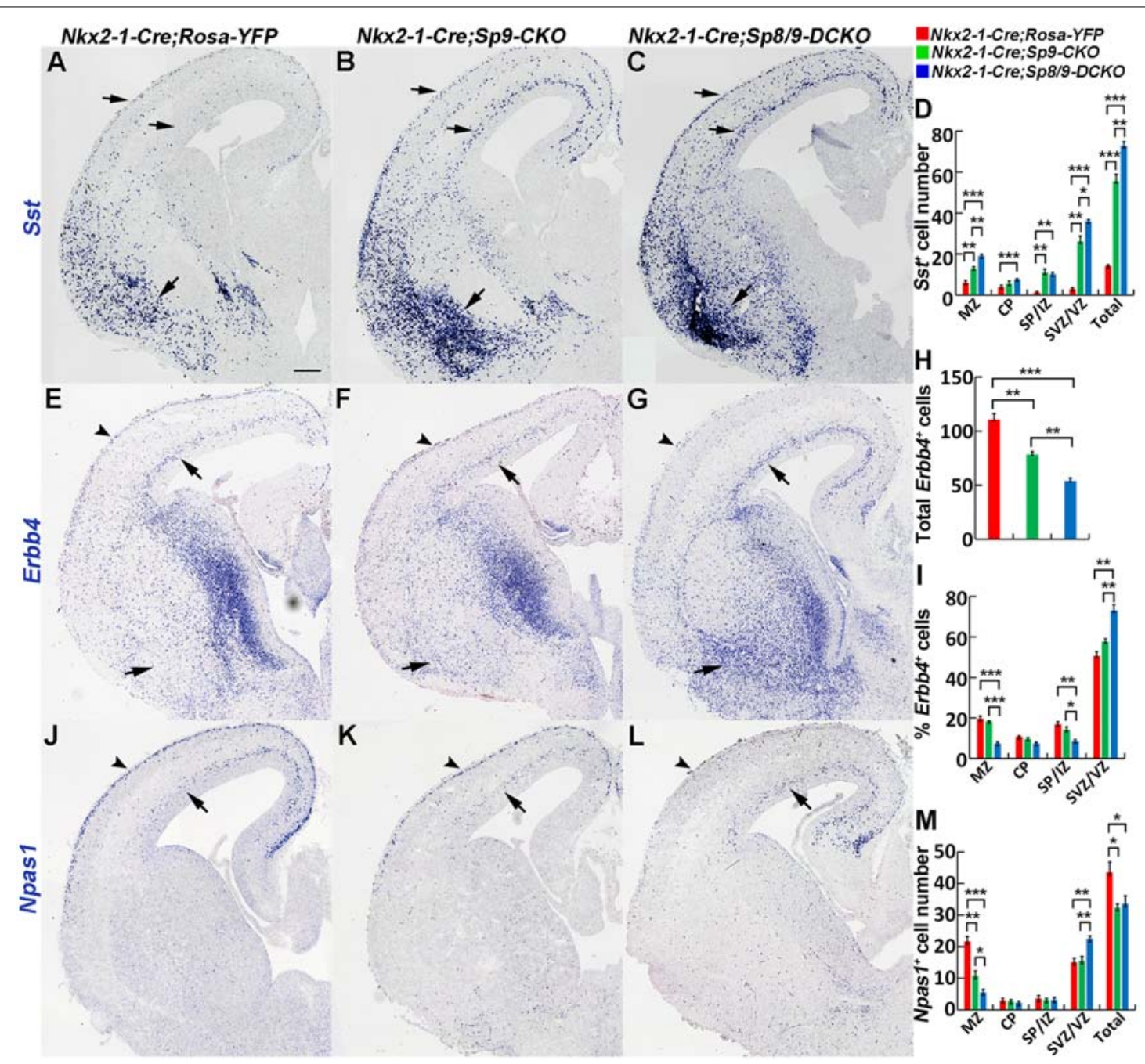

FIGURE 3 | In situ RNA hybridization of Sst, Erbb4 and Npas1. (A-D) Sst mRNA expression was increased in the E15.5 neocortex (arrows) and ventral telencephalon (arrows) of mutants. More Sst ${ }^{+}$cells were found in the ventral telencephalon of double mutants than single mutants and controls (arrows).

(E-I) Abnormal migration of Erbb4 ${ }^{+}$cells (PV+ immature cortical interneurons) in mutant mice. Double mutants appeared to have relatively more Erbb4 ${ }^{+}$cells in the cortical SVZ (arrows) and in the ventral telencephalon (arrows) and relatively fewer Erbb4 ${ }^{+}$cells in the cortical marginal zone (MZ; arrowheads). (J-M) Decreased Npas $1^{+}$cells in the cortical MZ (arrowheads) and increased Npas $1^{+}$cells in the cortical SVZ (arrows) were observed in double mutants compared to single mutants and controls. ${ }^{*} P<0.05 ;{ }^{* *} P<0.01 ;{ }^{* * *} P<0.001$. Scale bar: $200 \mu \mathrm{m}$ in (A) for (A-C,E-G,J-L).

increase in $\mathrm{NKX} 2-1^{+} / \mathrm{SP}^{+}$cells compared to wild type controls (Figures $\mathbf{1} \mathbf{M}, \mathbf{N}$ ). Given a high degree of similarity between the $S p 8$ and $S p 9$ genes, upregulation of SP8 expression may compensate for $S p 9$ function in the MGE. To test this hypothesis, we compared MGE development in control, Nkx2-1-Cre; Sp9CKO (Sp9 single mutant) and Nkx2-1-Cre; Sp8/9-DCKO (Sp8/9 double mutant) mice.

\section{Nkx2-1-Cre; Sp8/9-DCKO Mice Show Severe Defects in Tangential Migration of MGE-Derived Cortical Interneurons}

In E13.5 Nkx2-1-Cre; Rosa-YFP control mice, many $\mathrm{GFP}^{+}$ cells entered the medial region of the cortex (Figures $\mathbf{2 A}, \mathbf{A}^{\prime}$ ). In Nkx2-1-Cre; Sp9-CKO single mutants, $\mathrm{GFP}^{+}$cells also migrated to the cortex, although less efficiently (Figures $\mathbf{2 B}, \mathbf{B}^{\prime}$; Liu et al., 2018). However, in Nkx2-1-Cre; Sp8/9-DCKO double mutants, $\mathrm{GFP}^{+}$cells only reached the lateral cortex (Figures $2 \mathrm{C}, \mathrm{C}^{\prime}$ ). We also found few $\mathrm{GFP}^{+}$cells in the $\mathrm{LGE}$ SVZ of single and double mutants (Figures $\mathbf{2} \mathbf{B}^{\prime \prime}, \mathbf{C}^{\prime \prime}$ ), whereas a subpopulation of $\mathrm{GFP}^{+}$cells were observed in the LGE $\mathrm{VZ} / \mathrm{SVZ}$ that tangentially migrated to the cortex of control mice (Figure 2A").

At E15.5, in control, Nkx2-1-Cre; Sp9-CKO and Nkx2-1-Cre; Sp8/9-DCKO mice, migration of cortical interneurons appears to occur primarily in two streams, in the cortical MZ and the SVZ, but Nkx2-1-Cre; Sp9-CKO and Nkx2-1-Cre; Sp8/9-DCKO mice had fewer $\mathrm{GFP}^{+}$cells in the cortex (Figures $\left.2 \mathrm{D}-\mathbf{F}^{\prime \prime}, \mathbf{G}\right)$. We then analyzed the distribution of $\mathrm{MGE}$-derived $\left(\mathrm{GFP}^{+}\right)$cortical interneurons. The number of $\mathrm{GFP}^{+}$cells in the MZ, CP, SP, 


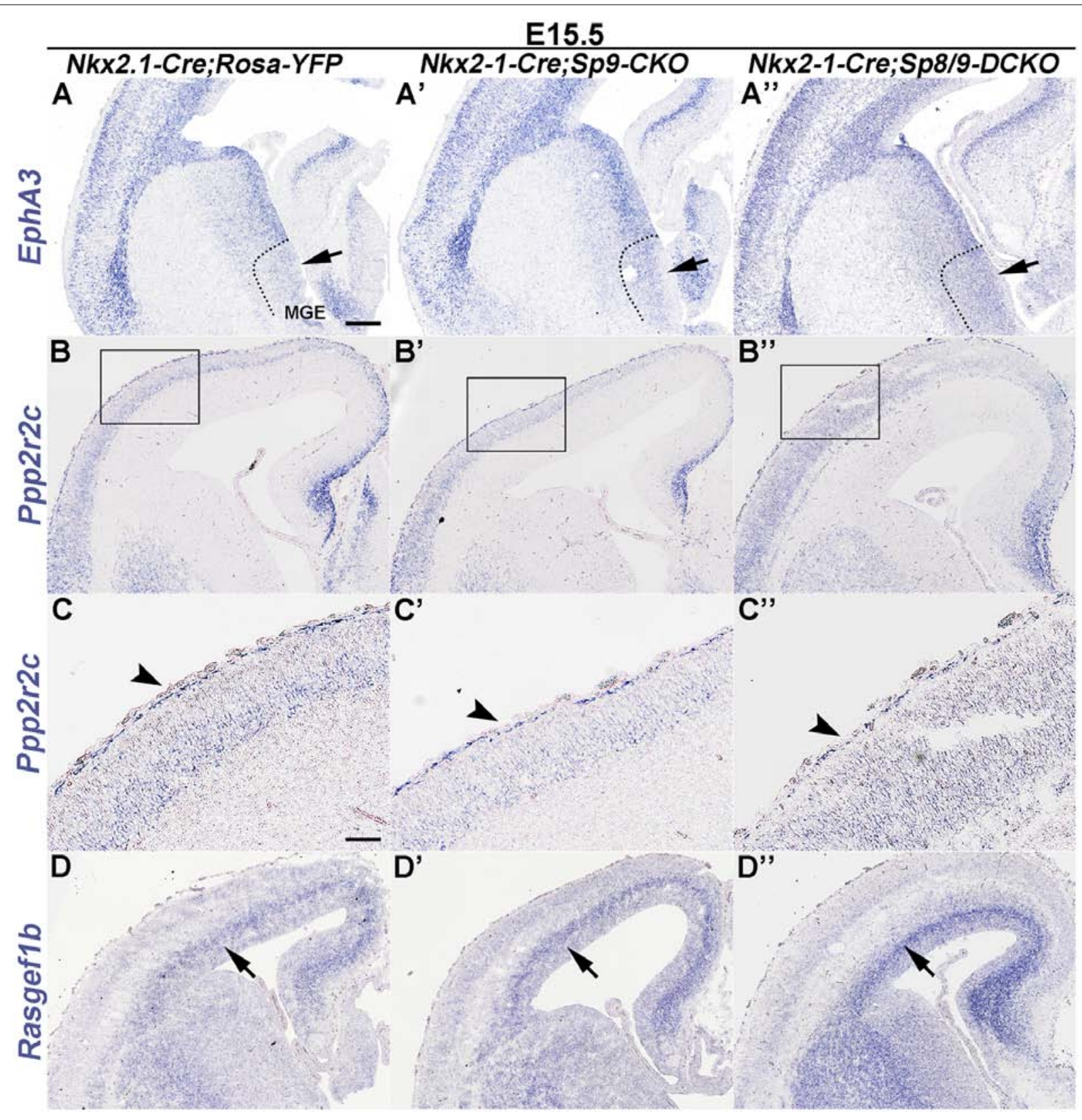

FIGURE 4 | In situ RNA hybridization of Epha3, Ppp2r2c and Rasgef1b in coronal hemisections at E15.5. (A-A") EphA3 was upregulated in the mutant MGE (arrows). (B-C') Ppp2r2c expression was reduced in the mutant cortical MZ (arrowheads). (D-D") Rasgef1b expression was increased in the mutant cortical SVZ (arrows). Note that dysregulation of these genes was more prominent in Sp8/9 double mutants than in Sp9 single mutants. Scale bars: $200 \mu \mathrm{m}$ in (A) for (A-B",D-D"); $100 \mu \mathrm{m}$ in (C) for (C-C").

IZ and SVZ of the cortex were greatly reduced in single and double mutants compared with controls (Figure 2G), as was the percentage of $\mathrm{GFP}^{+}$cells in the SP and the IZ (Figure $2 \mathrm{H}$ ). In contrast, the percentage of mutant $\mathrm{GFP}^{+}$cells in the cortical SVZ was increased (Figure $2 \mathrm{H}$ ). Notably, the percentage of $\mathrm{GFP}^{+}$ cells was notably higher in the SVZ of Sp8/9 double mutants than in $S p 9$ single mutants, and the percentage of $\mathrm{GFP}^{+}$cells in the SP and the IZ was much lower (Figure $2 \mathbf{H}$ ). Again, very few $\mathrm{GFP}^{+}$cells were found in the LGE SVZ of mutants
(Figures $2 \mathrm{D}^{\prime \prime}-\mathrm{F}^{\prime \prime}$ ). The ectopic accumulation of MGE-derived $\mathrm{GFP}^{+}$cells was observed in Nkx2-1-Cre; Sp9-CKO mice (Liu et al., 2018), but the loss of both $S p 8$ and $S p 9$ function led to severe increases in $\mathrm{GFP}^{+}$cells in the ventral telencephalon (Figures 2D-F). Taken together, both $S p 9$ and $S p 8$ promote the tangential migration of MGE-derived cortical interneurons from the MGE to the cortex and control the migration of cortical interneurons that follow distinct pathways in the cortex. In addition, loss of $S p 8$ function in $S p 9$ mutants further 


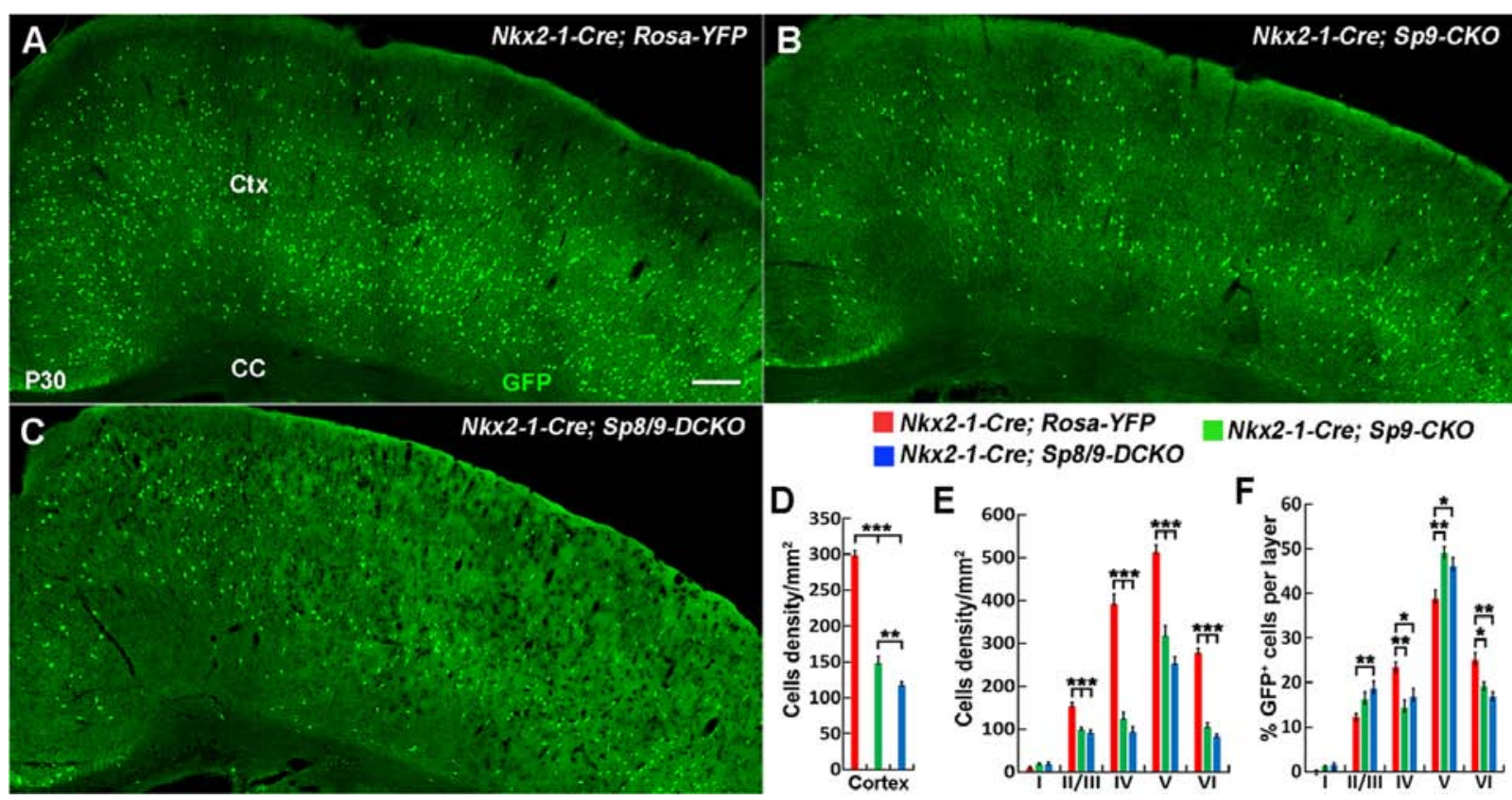

FIGURE 5 | The number of MGE-derived cortical interneurons was significantly reduced in double mutants compared with single mutants and controls at P30. (A-C) GFP+ cells in the cortex of Nkx2-1-Cre; Rosa-YFP (control), Nkx2-1-Cre; Sp9F/F; Rosa-YFP (single mutant) and Nkx2-1-Cre; Sp8F/F; Sp9F/F; Rosa-YFP (double mutant) mice. (D-F) Quantified data showed that the density of GFP+ cells in the somatosensory cortex was reduced in single mutants and further reduced in double mutants compared with controls. Ctx, cortex; CC, corpus callosum. ${ }^{*} P<0.05 ;{ }^{* *} P<0.01 ;{ }^{* * *} P<0.001$. Scale bars: $200 \mu \mathrm{m}$ in (A) for (A-C).

increased E15.5 cortical interneurons in the deep migration zone (SVZ).

\section{Molecular Defects in Tangentially Migrating Sp8/9 Double Mutant MGE-Derived Cortical Interneurons}

We next examined the molecular profile of tangentially migrating cells in these mutants at E15.5. We previously demonstrated using RNA-Seq and ChIP-Seq (chromatin coimmunoprecipitation followed by high-throughput DNA sequencing) that $S p 9$ has a curial transcriptional role in regulating MGE-derived cortical interneuron development (Liu et al., 2018). Loss of $S p 9$ function resulted in upregulation of Sst expression in a subset of MGE-derived cells; however, Sst expression was further upregulated in $S p 8 / 9$ double mutants (Figures 3A-C). Indeed, although $S p 8 / 9$ double mutants and $S p 9$ single mutants had almost same numbers of $\mathrm{GFP}^{+}$ cells in the cortex at E15.5 (Figure 2G), double mutants had many more $S s t^{+}$cells than $S p 9$ single mutants (Figure 3D). Moreover, more ectopic Sst $t^{+}$cells accumulated in the ventral telencephalon in double mutants compared with single mutants (Figures 3B,C). Erbb4 is mainly expressed in migratory and mature $\mathrm{PV}^{+}$cortical interneurons (Fazzari et al., 2010; Mayer et al., 2018). We found that the percentage of Erbb4 ${ }^{+}$cells was reduced in the mutant cortical MZ, whereas it was greatly increased in the cortical SVZ (Figures 3E-I). Again, Nkx21-Cre; Sp8/9-DCKO mice had relative more Erbb4 ${ }^{+}$cells in the cortical SVZ and in the ventral telencephalon than $N k \times 2$ -
1-Cre; Sp9-CKO mice (Figures 3E-I). This finding suggests that loss of $S p 8 / 9$ function results in relatively more immature $\mathrm{PV}^{+}$cortical interneurons that migrate in the cortical SVZ and that ectopically accumulate in the ventral telencephalon. Npas1 is mainly expressed in cortical interneurons in the MZ, although a small number of interneurons in the cortical SVZ also express Npas1 (Cobos et al., 2006; Stanco et al., 2014). We found that the percentage of $\mathrm{Npas}^{+}$cells in the cortical $\mathrm{MZ}$ were reduced, whereas it was greatly increased in the cortical SVZ of mutants compared with controls (Figures 3J-M).

Epha3 expression was increased in the mutant MGE at E15.5 (Figures $\mathbf{4 A}-\mathbf{A}^{\prime \prime}$ ), which explained why the LGE had very few MGE-derived cells, as enhanced Eph/ephrin signaling in the LGE VZ/SVZ increases the repulsive effect on migrating interneurons (Zimmer et al., 2008; Rudolph et al., 2010; Villar-Cerviño et al., 2015; Liu et al., 2018). Intracellular signaling molecule $P p p 2 r 2 c$ is mainly expressed in cortical interneurons in the MZ, whereas Rasgeflb is mainly expressed in cortical interneurons in the SVZ (Colasante et al., 2009; Antypa et al., 2011; Friocourt and Parnavelas, 2011). We found that the expression of Ppp2r2c in the cortical MZ was reduced, whereas the expression of Rasgef $1 b$ was greatly increased in the cortical SVZ of mutants compared with controls (Figures 4B-D"'; Antypa et al., 2011). Notably, these phenotypes were more prominent in $S p 8 / 9$ double mutants than in $S p 9$ single mutants (Figures $\mathbf{4} \mathbf{A}-\mathbf{D}^{\prime \prime}$ ), further suggesting that $S p 8$ supplements the role of $S p 9$ in regulating MGE-derived cortical interneuron migration. 


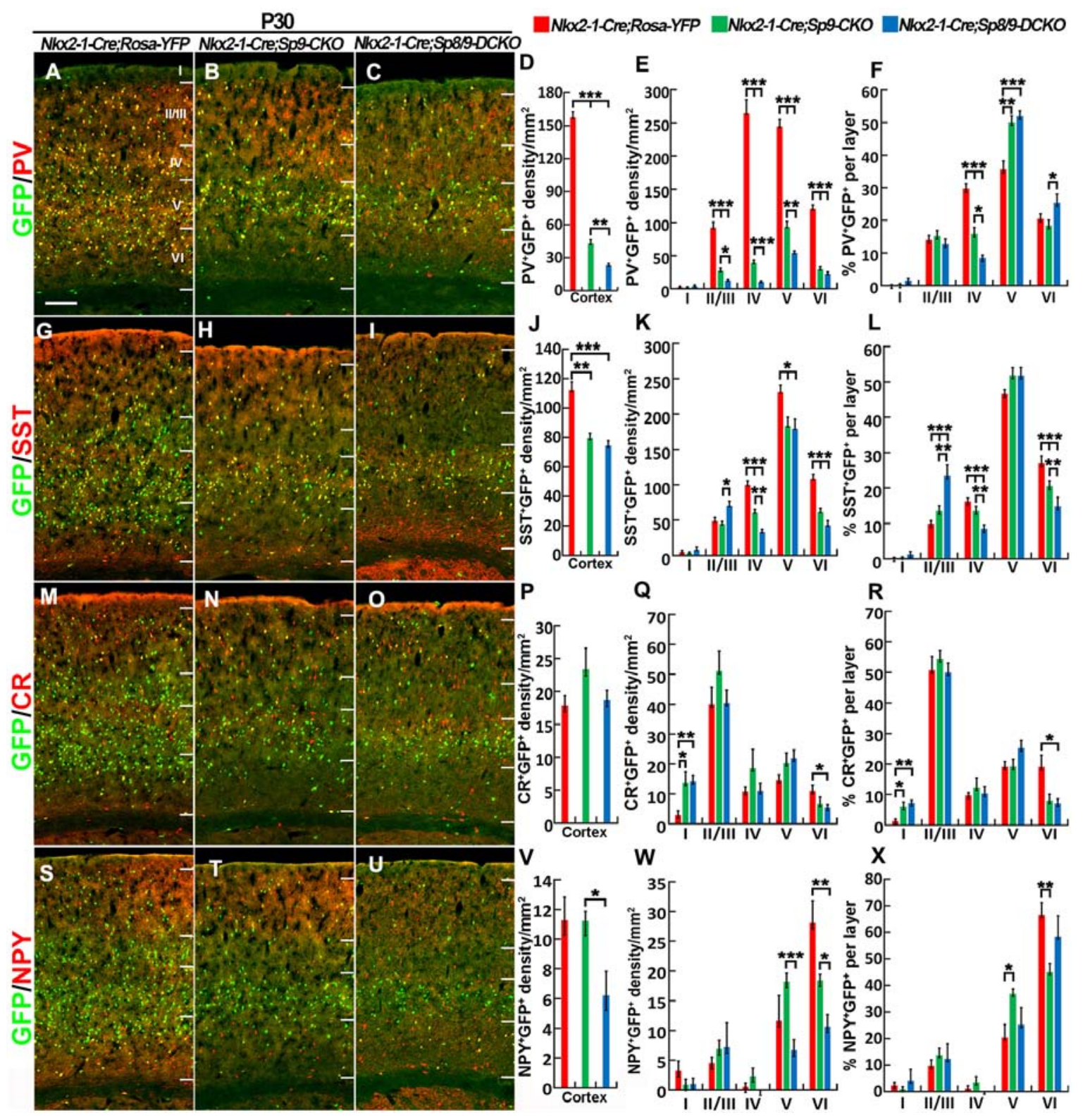

FIGURE 6 | PV+ cortical interneurons are severely reduced in the Sp8/9 double mutant cortex at P30. (A-C,G-I,M-O,S-U) GFP and cortical interneuron markers [PV, SST, calretinin (CR) and NPY] in double-immunostained coronal sections of the somatosensory cortex. (D-F,J-L,P-R,V-X) Quantified data showed that the density of $\mathrm{PV}^{+} / \mathrm{GFP}^{+}$cells was severely reduced in double mutants compared with single mutants and controls. The density of $\mathrm{SST}^{+} / \mathrm{GFP}^{+}$and $\mathrm{NPY}^{+} / \mathrm{GFP}^{+}$cells was also reduced in the mutant somatosensory cortex, whereas $\mathrm{CR}^{+} / \mathrm{GFP}+$ cells were less affected. ${ }^{*} P<0.05 ;{ }^{* *} P<0.01 ;{ }^{* * *} P<0.001$. Scale bars: $200 \mu \mathrm{m}$ in (A) for (A-C,G-I,M-O,S-U).

\section{PV $^{+}$Cortical Interneurons Are Severely Reduced in Sp8/Sp9 Double Mutants}

Analysis of the P30 neocortex showed profound MGE-derived cortical interneuron defects, especially $\mathrm{PV}^{+}$cortical interneurons. We found that the density of MGE-derived cortical interneurons $\left(\mathrm{GFP}^{+}\right.$cells) in the somatosensory cortex was significantly reduced in Nkx2-1-Cre; Sp8/9-DCKO mice compared within Nkx2-1-Cre; Sp9-CKO mice and controls (Figures 5A-D). The density of $\mathrm{GFP}^{+}$cells was also greatly reduced in each cortical layer (Figure 5E), but the percentage of $\mathrm{GFP}^{+}$cells in cortical layers II/III and V was relatively increased in single and double mutants (Figure 5F).

We next quantified the density of $\mathrm{GFP}^{+} / \mathrm{PV}^{+}, \mathrm{GFP}^{+} / \mathrm{SST}^{+}$, $\mathrm{GFP}^{+} / \mathrm{NPY}^{+}$, and $\mathrm{GFP}^{+} / \mathrm{CR}^{+}$interneurons in the somatosensory cortex (Figures 6A-X). The most prominent phenotype was severe loss of $\mathrm{GFP}^{+} / \mathrm{PV}^{+}$cortical interneurons: Sp8/9 double mutants showed $\sim 80 \%$ reduction, and Sp9 single mutants showed $\sim 65 \%$ reduction (Figures $6 \mathrm{~A}-\mathrm{E}$ ). $\mathrm{GFP}^{+} / \mathrm{NPY}^{+}$ 


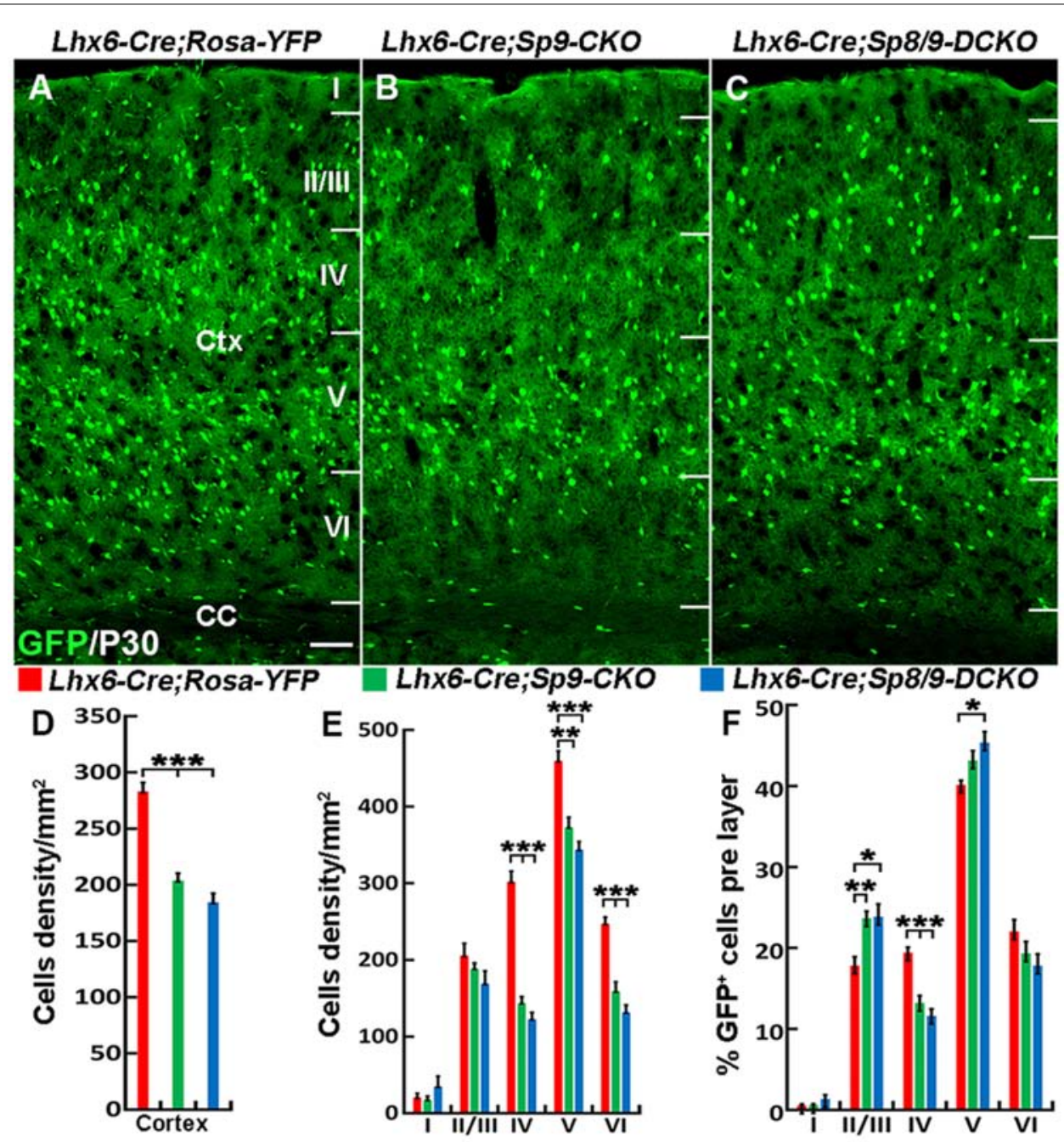

FIGURE 7 | The number of MGE-derived cortical interneurons is greatly reduced in the somatosensory cortex of Lhx6-Cre; Sp8 ${ }^{F / F}$; Sp9F/F; Rosa-YFP mice compared with Lhx6-Cre; Rosa-YFP and Lhx6-Cre; Sp9F/F; Rosa-YFP mice at P30. (A-C) GFP ${ }^{+}$cells in the somatosensory cortex. (D-F) Quantified data from the above experiments. The density of GFP+ ${ }^{+}$cells was significantly reduced in mutants compared with controls. Ctx, cortex; CC, corpus callosum. ${ }^{*}<0.05$; ${ }^{* *} P<0.01 ;{ }^{* * *} P<0.001$. Scale bars: $200 \mu \mathrm{m}$ in $(\mathbf{A})$ for $(\mathbf{A}-\mathbf{C})$.

cortical interneurons were also reduced in double mutants compared with single mutants or controls (Figures 6S-X), whereas $\mathrm{GFP}^{+} / \mathrm{SST}^{+}$cells and $\mathrm{GFP}^{+} / \mathrm{CR}^{+}$cells were less affected in double mutants (Figures 6G-K,M-R). In $S p 9$ single and $S p 8 / 9$ double mutants, the percentage of $\mathrm{GFP}^{+} / \mathrm{PV}^{+}$cells was higher in cortical layer $\mathrm{V}$, whereas the percentage of $\mathrm{GFP}^{+} / \mathrm{PV}^{+}$cells and $\mathrm{GFP}^{+} / \mathrm{SST}^{+}$cells was lower in cortical layer IV (Figures 6F,L), further indicating that $S p 8 / 9$ not only regulates migration but also affects the layer distribution of MGE-derived cortical interneurons.

Nkx2-1-Cre recombination occurs from the MGE VZ, the SVZ and the mantle zone ( $\mathrm{Xu}$ et al., 2008). We, therefore, used the Lhx6-Cre transgenic line to conditionally knock out Sp8/9 in postmitotic MGE-derived neurons (Fogarty et al., 2007; Nóbrega-Pereira et al., 2008) and quantified the density of $\mathrm{GFP}^{+}$, $\mathrm{GFP}^{+} / \mathrm{PV}^{+}$and $\mathrm{GFP}^{+} / \mathrm{SST}^{+}$interneurons in the somatosensory cortex. In general, loss of $S p 8 / 9$ function in postmitotic MGE cells resulted in greatly reduced $\mathrm{GFP}^{+}$and $\mathrm{GFP}^{+} / \mathrm{PV}^{+}$cortical interneuron density (Figures $7 \mathrm{~A}-\mathbf{F}, \mathbf{8 A}-\mathbf{L}$ ). Furthermore, an altered allocation of MGE-derived interneurons in the cortical layers was also observed in the mutants (Figures 7A-F, 8A-L), consistent with results of the Nkx2-1-Cre line knockouts.

\section{DISCUSSION}

The function of $S p 9$ in the MGE has been described (Liu et al., 2018), but the function of $S p 8$ in the MGE, which is highly homologous with $S p 9$ and weakly expressed in the MGE mantle zone, has not yet been studied. Here, we found that the expression of SP8 in the MGE was significantly increased in $S p 9$ null mutants. When we conditionally deleted both $S p 8$ and $S p 9$ in the MGE, the tangential migration of 


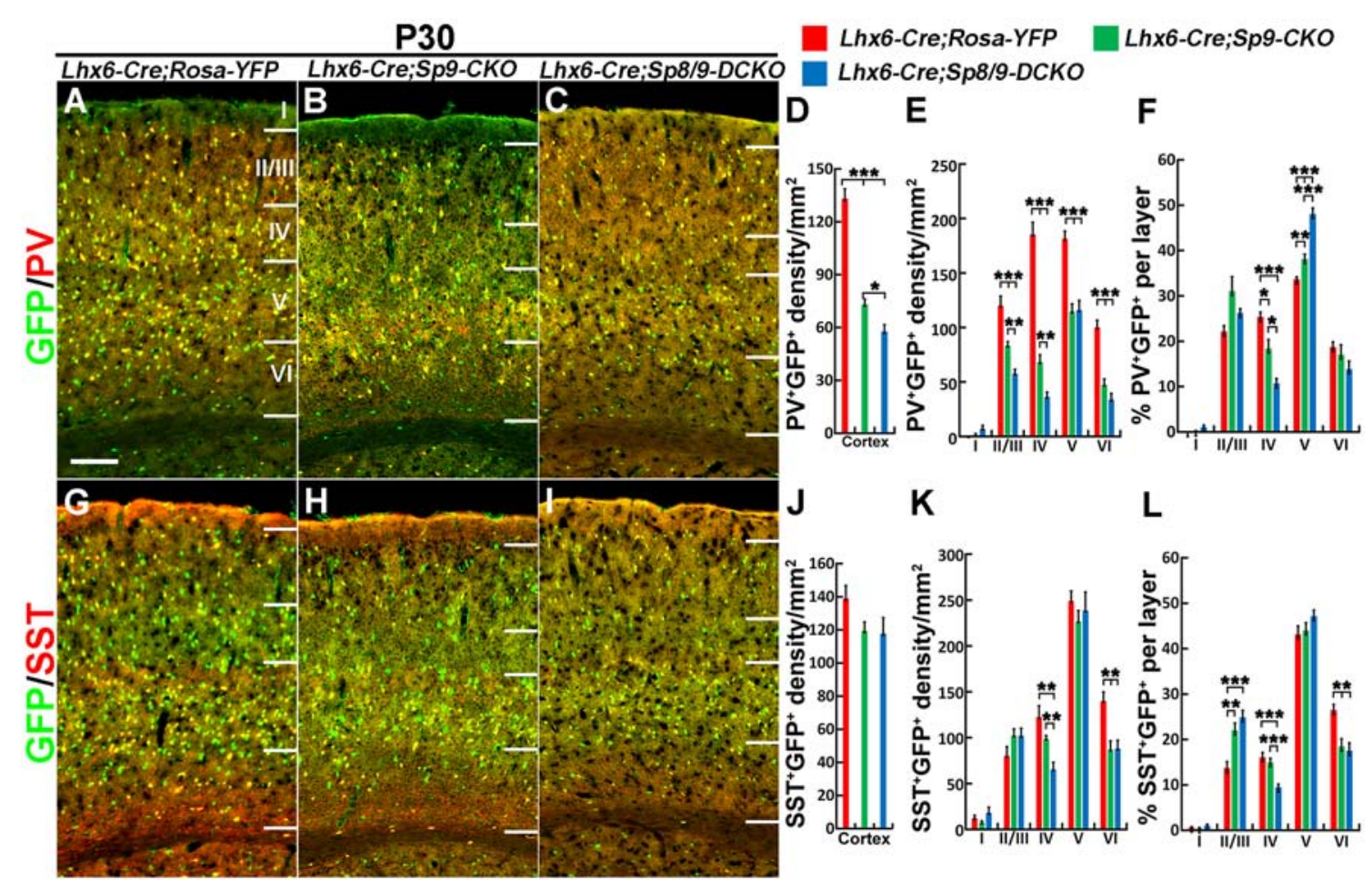

FIGURE $8 \mid \mathrm{PV}^{+}$cortical interneurons are severely reduced in the Lhx6-Cre; Sp8 $8^{\mathrm{F} / F}$; Spg F/F; Rosa-YFP somatosensory cortex. (A-C,G-I) GFP/PV and GFP/SST double-immunostained coronal sections. (D-F,J-L) The density of $\mathrm{PV}^{+} / \mathrm{GFP}+$ cells was severely reduced in double mutants compared with single mutants and controls, whereas the density of SST + GFP+ cells was less affected. ${ }^{*} P<0.05 ;{ }^{* *} P<0.01 ;{ }^{* * *} P<0.001$. Scale bars: $200 \mu \mathrm{m}$ in (A) for (A-C,G-I).

MGE-derived cortical interneurons was greatly inhibited, and the density of MGE-derived cortical interneurons, especially $\mathrm{PV}^{+}$ interneurons, was greatly reduced. These results suggest that $S p 8$ plays a supplementary role to $S p 9$ in regulating MGE-derived cortical interneuron development.

Previous studies have mainly focused on the function of $S p 8$ in the LGE, which show that $S p 8$ is important for the development of olfactory bulb interneurons and striatal medium spiny neurons (Waclaw et al., 2006; Liu et al., 2009; Li et al., 2011, 2018; Xu et al., 2018). SP8 is very weakly expressed in the MGE mantle zone (Ma et al., 2012; Vogt et al., 2014), but its function remains to be investigated. In the present study, we found that SP8 expression was upregulated in the Sp9 mutant MGE, indicating that SP8 could compensate for SP9 function. Indeed, when we used the Nkx2-1-Cre transgenic line to conditionally knock out $S p 8 / 9$, the tangential migration of MGE-derived cortical interneurons was blocked, resulting in ectopic accumulation of interneuron-like cells in the ventral telencephalon. We propose that the loss of $80 \%$ of $\mathrm{PV}^{+}$ cortical interneurons was mainly due to defects in interneuron migration, as conditional deletion of $S p 8 / 9$ in MGE neural stem cells using Nkx2-1-Cre lines and conditionally delete Sp8/9 in MGE-derived postmitotic cells using Lhx6-Cre lines show a similar reduction of cortex interneurons. Furthermore, we observed that the apoptotic cell death in the control, single and double mutant cortices were at the same levels (data not shown), suggesting that $S p 8 / 9$ do not affect the survival of MGE-derived cortical interneurons.

We suggest that this migratory deficit results from multiple mechanisms. In the double mutant neocortex, Erbb4 ${ }^{+}$immature $\mathrm{PV}$ interneurons mainly migrated in the cortical SVZ, whereas only a few migrated in the cortical MZ. Furthermore, we observed that more Erbb4 ${ }^{+}$cells ectopically accumulated in the ventral telencephalon. This finding explains why $\mathrm{PV}^{+}$cortical interneurons were severely reduced in the double mutant cortex compared with the single mutant cortex and indicate that $S p 8$ indeed has an important function in promoting MGE-derived cortical interneuron migration. The $P p p 2 r 2 c$ gene, encoding a subunit of protein phosphatase $2 \mathrm{~A}$, has a unique expression pattern in the embryonic mouse neocortex; interneurons in the cortical MZ express $P p p 2 r 2 c$, but interneurons in the cortical SVZ do not (Colasante et al., 2009; Antypa et al., 2011; Friocourt and Parnavelas, 2011). Rasgef1b expression appeared to be limited to the cortical SVZ interneuron stream (Colasante et al., 2009; Antypa et al., 2011; Friocourt and Parnavelas, 2011). The Rasgef $1 b$ gene encodes a guanine nucleotide exchange factor for Ras family proteins and is a downstream target of Arx (Friocourt and Parnavelas, 2011). We found that the expression of Rasgef $1 b$ was significantly upregulated after removal of $S p 8 / 9$, whereas the expression of $P p p 2 r 2 c$ in the cortical MZ was significantly decreased. The dysregulation of these two genes may explain the great increase in the proportion of MGE-derived $\mathrm{GFP}^{+}$cells 
in the cortical SVZ. Notably, loss of Sp8/9 in the dorsal LGE also induced a block in tangential migration from the LGE to the olfactory bulb (Li et al., 2018; Guo et al., 2019). Thus, we propose that $S p 8 / 9$ genes might play a general role in regulating the tangential migration of telencephalic interneurons.

\section{AUTHOR CONTRIBUTIONS}

GT and ZL performed all experiments and analyses. YW, XS, SW and HD helped conduct some experiments and analyze the data.

\section{REFERENCES}

Antypa, M., Faux, C., Eichele, G., Parnavelas, J. G., and Andrews, W. D. (2011). Differential gene expression in migratory streams of cortical interneurons. Eur. J. Neurosci. 34, 1584-1594. doi: 10.1111/j.1460-9568.2011.07896.x

Bell, S. M., Schreiner, C. M., Waclaw, R. R., Campbell, K., Potter, S. S., and Scott, W. J. (2003). Sp8 is crucial for limb outgrowth and neuropore closure. Proc. Natl. Acad. Sci. U S A 100, 12195-12200. doi: 10.1073/pnas.2134 310100

Cobos, I., Long, J. E., Thwin, M. T., and Rubenstein, J. L. (2006). Cellular patterns of transcription factor expression in developing cortical interneurons. Cereb. Cortex 16, i82-i88. doi: 10.1093/cercor/bhk003

Colasante, G., Sessa, A., Crispi, S., Calogero, R., Mansouri, A., Collombat, P., et al. (2009). Arx acts as a regional key selector gene in the ventral telencephalon mainly through its transcriptional repression activity. Dev. Biol. 334, 59-71. doi: 10.1016/j.ydbio.2009.07.014

Del Pino, I., Rico, B., and Marin, O. (2018). Neural circuit dysfunction in mouse models of neurodevelopmental disorders. Curr. Opin. Neurobiol. 48, 174-182. doi: $10.1016 /$ j.conb.2017.12.013

Fazzari, P., Paternain, A. V., Valiente, M., Pla, R., Luján, R., Lloyd, K., et al. (2010). Control of cortical GABA circuitry development by $\mathrm{Nrg} 1$ and ErbB 4 signalling. Nature 464, 1376-1380. doi: 10.1038/nature08928

Fogarty, M., Grist, M., Gelman, D., Marín, O., Pachnis, V., and Kessaris, N. (2007). Spatial genetic patterning of the embryonic neuroepithelium generates GABAergic interneuron diversity in the adult cortex. J. Neurosci. 27, 10935-10946. doi: 10.1523/JNEUROSCI.1629-07.2007

Friocourt, G., and Parnavelas, J. G. (2011). Identification of Arx targets unveils new candidates for controlling cortical interneuron migration and differentiation. Front. Cell. Neurosci. 5:28. doi: 10.3389/fncel.2011.00028

Gelman, D. M., and Marín, O. (2010). Generation of interneuron diversity in the mouse cerebral cortex. Eur. J. Neurosci. 31, 2136-2141. doi: 10.1111/j.14609568.2010.07267.x

Guo, T., Liu, G., Du, H., Wen, Y., Wei, S., Li, Z., et al. (2019). Dlx1/2 are central and essential components in the transcriptional code for generating olfactory bulb interneurons. Cereb. Cortex doi: 10.1093/cercor/bhz018 [Epub ahead of print].

Hansen, D. V., Lui, J. H., Flandin, P., Yoshikawa, K., Rubenstein, J. L., AlvarezBuylla, A., et al. (2013). Non-epithelial stem cells and cortical interneuron production in the human ganglionic eminences. Nat. Neurosci. 16, 1576-1587. doi: 10.1038/nn.3541

Hu, J. S., Vogt, D., Sandberg, M., and Rubenstein, J. L. (2017). Cortical interneuron development: a tale of time and space. Development 144, 3867-3878. doi: $10.1242 /$ dev.132852

Kriegstein, A., and Alvarez-Buylla, A. (2009). The glial nature of embryonic and adult neural stem cells. Annu. Rev. Neurosci. 32, 149-184. doi: 10.1146/annurev. neuro. 051508.135600

Li, X., Sun, C., Lin, C., Ma, T., Madhavan, M. C., Campbell, K., et al. (2011). The transcription factor Sp8 is required for the production of parvalbuminexpressing interneurons in the olfactory bulb. J. Neurosci. 31, 8450-8455. doi: 10.1523/JNEUROSCI.0939-11.2011

Li, J., Wang, C., Zhang, Z., Wen, Y., An, L., Liang, Q., et al. (2018). Transcription factors Sp8 and Sp9 coordinately regulate olfactory bulb interneuron development. Cereb. Cortex 28, 3278-3294. doi: 10.1093/cercor/bhx199
ZY and YY helped guide the project and discussed the results. ZY, ZX, YY and GT wrote and edited the manuscript.

\section{FUNDING}

This work was supported by a research grant to ZY from National Key Research and Development Program (973) of China (2018YFA0108000), National Natural Science Foundation of China (NSFC 31820103006, 31630032 and 31425011), and research grant to YY (NSFC 31700889).

Lim, L., Mi, D., Llorca, A., and Marín, O. (2018). Development and functional diversification of cortical interneurons. Neuron 100, 294-313. doi: 10.1016/j. neuron.2018.10.009

Liu, F., You, Y., Li, X., Ma, T., Nie, Y., Wei, B., et al. (2009). Brain injury does not alter the intrinsic differentiation potential of adult neuroblasts. J. Neurosci. 29, 5075-5087. doi: 10.1523/JNEUROSCI.0201-09.2009

Liu, Z., Zhang, Z., Lindtner, S., Li, Z., Xu, Z., Wei, S., et al. (2018). Sp9 regulates medial ganglionic eminence-derived cortical interneuron development. Cereb. Cortex doi: 10.1093/cercor/bhy133 [Epub ahead of print].

Long, J. E., Swan, C., Liang, W. S., Cobos, I., Potter, G. B., and Rubenstein, J. L. (2009). Dlxland2 and Mash1 transcription factors control striatal patterning and differentiation through parallel and overlapping pathways. J. Comp. Neurol. 512, 556-572. doi: 10.1002/cne.21854

Ma, T., Wang, C., Wang, L., Zhou, X., Tian, M., Zhang, Q., et al. (2013). Subcortical origins of human and monkey neocortical interneurons. Nat. Neurosci. 16, 1588-1597. doi: 10.1038/nn.3536

Ma, T., Zhang, Q., Cai, Y., You, Y., Rubenstein, J. L., and Yang, Z. (2012). A subpopulation of dorsal lateral/caudal ganglionic eminence-derived neocortical interneurons expresses the transcription factor Sp8. Cereb. Cortex 22, 2120-2130. doi: 10.1093/cercor/bhr296

Mayer, C., Hafemeister, C., Bandler, R. C., Machold, R., Batista Brito, R., Jaglin, X., et al. (2018). Developmental diversification of cortical inhibitory interneurons. Nature 555, 457-462. doi: 10.1038/nature25999

McKinsey, G. L., Lindtner, S., Trzcinski, B., Visel, A., Pennacchio, L. A., Huylebroeck, D., et al. (2013). Dlx1and2-dependent expression of Zfhxlb (Sip1, Zeb2) regulates the fate switch between cortical and striatal interneurons. Neuron 77, 83-98. doi: 10.1016/j.neuron.2012.11.035

Nóbrega-Pereira, S., Kessaris, N., Du, T., Kimura, S., Anderson, S. A., and Marín, O. (2008). Postmitotic Nkx2-1 controls the migration of telencephalic interneurons by direct repression of guidance receptors. Neuron 59, 733-745. doi: 10.1016/j.neuron.2008.07.024

Rubenstein, J. L., and Merzenich, M. M. (2003). Model of autism: increased ratio of excitation/inhibition in key neural systems. Genes Brain Behav. 2, 255-267. doi: 10.1034/j.1601-183x.2003.00037.x

Rudolph, J., Zimmer, G., Steinecke, A., Barchmann, S., and Bolz, J. (2010). Ephrins guide migrating cortical interneurons in the basal telencephalon. Cell Adh. Migr. 4, 400-408. doi: 10.4161/cam.4.3.11640

Srinivas, S., Watanabe, T., Lin, C. S., William, C. M., Tanabe, Y., Jessell, T. M., et al. (2001). Cre reporter strains produced by targeted insertion of EYFP and ECFP into the ROSA26 locus. BMC Dev. Biol. 1:4. doi: 10.1186/1471-213X-1-4

Stanco, A., Pla, R., Vogt, D., Chen, Y., Mandal, S., Walker, J., et al. (2014). NPAS1 represses the generation of specific subtypes of cortical interneurons. Neuron 84, 940-953. doi: 10.1016/j.neuron.2014.10.040

Villar-Cerviño, V., Kappeler, C., Nóbrega-Pereira, S., Henkemeyer, M., Rago, L., Nieto, M. A., et al. (2015). Molecular mechanisms controlling the migration of striatal interneurons. J. Neurosci. 35, 8718-8729. doi: 10.1523/JNEUROSCI. 4317-14.2015

Vogt, D., Hunt, R. F., Mandal, S., Sandberg, M., Silberberg, S. N., Nagasawa, T., et al. (2014). Lhx6 directly regulates Arx and CXCR7 to determine cortical interneuron fate and laminar position. Neuron 82, 350-364. doi: 10.1016/j. neuron.2014.02.030

Waclaw, R. R., Allen, Z. J. II., Bell, S. M., Erdélyi, F., Szabó, G., Potter, S. S., et al. (2006). The zinc finger transcription factor Sp8 regulates the generation and 
diversity of olfactory bulb interneurons. Neuron 49, 503-516. doi: 10.1016/j. neuron.2006.01.018

Wamsley, B., and Fishell, G. (2017). Genetic and activity-dependent mechanisms underlying interneuron diversity. Nat. Rev. Neurosci. 18, 299-309. doi: $10.1038 / \mathrm{nrn} .2017 .30$

Wonders, C. P., and Anderson, S. A. (2006). The origin and specification of cortical interneurons. Nat. Rev. Neurosci. 7, 687-696. doi: 10.1038/nrn1954

Xu, Z., Liang, Q., Song, X., Zhang, Z., Lindtner, S., Li, Z., et al. (2018). SP8 and SP9 coordinately promote D2-type medium spiny neuron production by activating Six3 expression. Development 145, dev165456. doi: 10.1242/dev. 165456

Xu, Q., Tam, M., and Anderson, S. A. (2008). Fate mapping Nkx2.1-lineage cells in the mouse telencephalon. J. Comp. Neurol. 506, 16-29. doi: 10.1002/cne.21529

Zhang, Q., Zhang, Y., Wang, C., Xu, Z., Liang, Q., An, L., et al. (2016). The zinc finger transcription factor Sp9 is required for the development of striatopallidal projection neurons. Cell Rep. 16, 1431-1444. doi: 10.1016/j.celrep.2016.06.090
Zimmer, G., Garcez, P., Rudolph, J., Niehage, R., Weth, F., Lent, R., et al. (2008). Ephrin-A5 acts as a repulsive cue for migrating cortical interneurons. Eur. J. Neurosci. 28, 62-73. doi: 10.1111/j.1460-9568.2008. 06320.x

Conflict of Interest Statement: The authors declare that the research was conducted in the absence of any commercial or financial relationships that could be construed as a potential conflict of interest.

Copyright (c) 2019 Tao, Li, Wen, Song, Wei, Du, Yang, Xu and You. This is an open-access article distributed under the terms of the Creative Commons Attribution License (CC BY). The use, distribution or reproduction in other forums is permitted, provided the original author(s) and the copyright owner(s) are credited and that the original publication in this journal is cited, in accordance with accepted academic practice. No use, distribution or reproduction is permitted which does not comply with these terms. 